\title{
A new twist of rubredoxin function in M. tuberculosis
}

Tatsiana Sushko ${ }^{1 \#}$, Anton Kavaleuski2\#, Irina Grabovec ${ }^{2}$, Anna Kavaleuskaya ${ }^{2}$, Daniil Vakhrameev $^{3}$, Sergei Bukhdruker ${ }^{3}$, Egor Marin $^{3}$, Alexey Kuzikov ${ }^{4,5}$, Rami Masamrekh ${ }^{4,5}$, Larisa V. Sigolaeva ${ }^{4,6}$, Victoria Shumyantseva ${ }^{4,5}$, Kouhei Tsumoto ${ }^{1,7}$, Valentin Borshchevskiy ${ }^{3}$, Andrei Gilep $^{2}$, and Natallia Strushkevich ${ }^{8 *}$.

1 - The Institute of Medical Science, the University of Tokyo, Tokyo, Japan

2 - Institute of Bioorganic Chemistry, National Academy of Sciences of Belarus, Minsk, Belarus.

3 - Research Center for Molecular Mechanisms of Aging and Age-Related Diseases, Moscow, Institute of Physics and Technology (MIPT), Dolgoprudny, Russia.

4 - Institute of Biomedical Chemistry, Moscow, Russia

5 - Pirogov Russian National Research Medical University, Moscow, Russia

6 - Department of Chemistry, M.V. Lomonosov Moscow State University, 119991 Moscow,

Russia

7 - Department of Bioengineering, School of Engineering, the University of Tokyo, Tokyo, Japan.

8 - Skolkovo Institute of Science and Technology, Moscow, Russia.

\# - authors equally contributed to this work

*- to whom correspondence should be addressed: N.Strushkevich@skoltech.ru

Natallia Strushkevich, 121205 Skolkovo Institute of Science and Technology, Bolshoy

Boulevard 30, bld. 1, room G11-2-21, Moscow, Russia; tel. +7 985-699-1410

Running title: RubB supports electron transfer to CYPs in Mtb

Abbreviations: CYP - cytochrome P450, RubB - rubredoxin B, Mtb - Mycobacterium tuberculosis, SPE - screen-printed electrode, MWCNT - multiwalled carbon nanotubes, FprA NADPH-ferredoxin reductase, FdR - NADH- ferredoxin reductase, Arh1 - adrenodoxin reductaselike flavoprotein, PETH - ferredoxin - NADP reductase, ITC - isothermal calorimetry, CD circular dichroism, DSC - differential scanning calorimetry, HPLC - high performance liquid chromatography, RMSD - root mean square deviation.

Enzymes: CYP142 (EC 1.14.15.28), CYP124 (EC 1.14.15.14), CYP125 (EC 1.14.15.29), NADPH-ferredoxin reductase FprA (EC 1.18.1.2), NADH-ferredoxin reductase FdR (EC 1.18.1.3), adrenodoxin reductase-like flavoprotein Arh1 (EC 1.18.1.2), ferredoxin - NADP reductase PETH (EC 1.18.1.2).

Databases: Atomic coordinates and structure factors were deposited in the Protein Data Bank (www.rcsb.org) under accession code PDB ID: 7A9A.

Key words: rubredoxin, $M$. tuberculosis, electron transfer, cytochrome P450, CYP, redox partner

Conflict of interests: The authors declare no conflict of interest. 


\begin{abstract}
Electron transfer mediated by metalloproteins drives many biological processes. Rubredoxins are ubiquitous iron-containing electron carriers that play important roles in bacterial adaptation to changing environmental conditions. In Mycobacterium tuberculosis, oxidative and acidic stresses as well as iron starvation induce rubredoxin expression. However, their functions during $M$. tuberculosis infection is unknown. In the present work, we show that rubredoxin B (RubB) supports catalytic activity of mycobacterial cytochrome P450s, CYP124, CYP125, and CYP142, which are important for bacterial viability and pathogenicity. We solved the crystal structure of RubB and characterized the interaction between RubB and CYPs using site-directed mutagenesis. Mutations that neutralized single charge on the surface of RubB did not dramatically decrease activity of studied CYPs, and isothermal calorimetry (ITC) experiments indicated that interactions are transient and not highly specific. Our findings suggest that a switch from ferredoxins to rubredoxins support CYP activity in M. tuberculosis-infected macrophages. Our electrochemical experiments suggest potential applications of RubB in biotechnology.
\end{abstract}

\title{
Introduction
}

Tuberculosis was the first infectious disease to be declared a global health emergency by the World Health Organization; tuberculosis causes more than 1,7 million deaths every year. Multiple mechanisms allow Mycobacterium tuberculosis (Mtb), the bacterium that causes tuberculosis, to survive within macrophages. These mechanisms include production of the antioxidant molecule mycothiol [1], activation of the catalase-peroxidase katG and superoxide dismutases sodA and sodC [2], and synthesis of truncated hemoglobins [3]. In phagosomes, Mtb resides in an acidic environment, thus requiring mechanisms to sustain growth under oxidative and acidic stresses. Moreover, during granuloma formation Mtb experiences drastic iron deprivation that influences the intensity of iron-sulfur clusters assembly in redox proteins [4]. The presence of a variety of genes that encode $[3 \mathrm{Fe}-4 \mathrm{~S}]$ and $[4 \mathrm{Fe}-4 \mathrm{~S}]$ ferredoxins and rubredoxins identified in Mtb suggests the important role of iron-containing proteins in maintaining redox homeostasis. We hypothesized that rubredoxins, which have been suggested to be part of an evolutionary chain between ferredoxins and flavodoxins [5], might be important in reactions catalyzed by cytochrome P450 (CYP) proteins.

Rubredoxins are small ( $\sim 6 \mathrm{kDa})$ iron-sulfur proteins that are crucial for oxidative stress responses. They rapidly transfer metabolic reducing equivalents to oxygen or reactive oxygen species and act as electron carriers in many biochemical pathways. The Mtb genome contains a highly conserved operon comprised of two tandemly arranged rubredoxin-encoding genes, RubA (Rv3251c) and $R u b B$ (Rv3250c), and the gene alkB (Rv3252c), which encodes an alkane hydroxylase. The gene products are probably involved in alkane and fatty acid metabolism. Our bioinformatic analysis of rubredoxins across different phylogenetic groups (Fig.S1, S2) showed that $R u b A$ is conserved in Actinobacteria and is specific for this family, whereas $R u b B$ is observed in gamma- and betaproteobacteria. Large and distinctive rubredoxin families are found in Bacteroidetes and 
Firmicutes phyla; these genes group with genes encoding primitive archaeal rubredoxins, and plant-specific rubredoxins, demonstrating evolutionary conservation of rubredoxin scaffold.

Expression of both $\mathrm{Mtb}$ rubredoxins is induced under iron starvation [4] and during iron chelator 2,2'-bipyridyl application [6]. Oxidative stress induced by the alkaloid ascidemin or nitrosative stress caused by diethylenetriamine/NO or a mixture of S-nitrosoglutathione and potassium cyanide strongly induce expression of rubredoxins in Mtb culture. Finally, rubredoxins are also induced by culture of $\mathrm{Mtb}$ in acidic $\mathrm{pH}[6,7]$. Notably, rubredoxin induction is often accompanied by inhibition of ferredoxin expression.

Induction of rubredoxin expression during oxidative and acidic stresses as well as under iron starvation might be due to the physical-chemical properties of rubredoxins. Rubredoxins need only one iron ion, which is beneficial during residence within macrophages in which microelements are deficient. Further, rubredoxins are usually of lower molecular mass than ferredoxins and do not require scaffold proteins, thus requiring few biosynthetic resources than ferredoxins. Moreover, rubredoxin activity is not inhibited by oxidative, acidic and temperature stresses, which may be crucial in the face of a host immune response $[8,9]$. This evidence suggests that rubredoxins more advantageous redox partners for CYPs than ferredoxins.

To test this idea, we purified RubB protein and measured enzymatic activity of three Mtb CYPs in the reconstituted system, containing RubB and different reductases as redox-partners. Our results demonstrate that RubB supports CYP-dependent catalysis. We also performed biophysical characterization of RubB including crystal structure determination. Site-directed mutagenesis was used for mapping of the protein-protein interactions within the RubB - CYP complex. Based on data obtained using purified RubB mutants, we suggest that the RubB interaction with CYPs is transient and not highly specific, as point charge neutralization on the surface of RubB does not dramatically affect CYP activity. Overall, our results provide new insights into electron transfer within different classes of redox proteins in $\mathrm{Mtb}$ and suggest that these interactions might be important switch mechanisms during different stages of Mtb infection.

\section{Results}

\section{Cloning, expression and purification of RubB}

$R u b B$ was cloned into the pET11a vector and overexpressed in Escherichia coli. According to a previous report, both iron and zinc-substituted rubredoxins are produced during heterologous expression in E.coli [10]. As binding of zinc and iron to RubB might be a competitive process [11], we provided extra iron during culture to obtain primarily the iron-containing form. RubB was purified to a homogenous state using sequential ion-exchange and size-exclusion chromatography. Based on size-exclusion chromatography, RubB was predominantly in the monomeric form and had spectral properties typical of oxidized Fe-S rubredoxins with maxima at 280, 380, and $490 \mathrm{~nm}$ (Fig. 1). 


\section{Thermal stability of RubB}

Some rubredoxins are hyper thermostable [12]. Previous circular dichroism (CD) studies of zincsubstituted Mtb RubB revealed that RubB is stable to $80^{\circ} \mathrm{C}$; however, $26 \%$ of residues of that protein are in the unstructured N-terminal tag, and its unfolding temperature was not determined [10]. Our analysis of thermal stability of purified RubB from Mtb using differential scanning calorimetry (DSC) revealed no peaks indicative of thermal unfolding in the temperature range 10$110{ }^{\circ} \mathrm{C}$ over the $\mathrm{pH}$ range from 6 to 7.4 (Table 1). A constant increase in heat capacity due to hydration of residues was observed over this temperature range. Thermal stability at acidic $\mathrm{pH}$, conditions under which protein side chain are protonated, was more stable at $\mathrm{pH} 5$ (melting temperature $\left(\mathrm{T}_{\mathrm{m}}\right)$ of $\left.87.2^{\circ} \mathrm{C}\right)$ than at higher or low $\mathrm{pH}$ (Table 1). The CD-spectra of RubB measured at $\mathrm{pH}$ values from 5.0 to 7.4 were not significantly different. Thus, RubB has a native fold and high thermal stability in the $\mathrm{pH}$ range ( $\mathrm{pH}$ 4.5-6.2) of the phagosomes of macrophages where Mtb resides [9]. Our data are in good correlation with heat-induced denaturation of rubredoxins from other sources [13].

\section{Electrochemistry}

Rubredoxins are involved in the electron transfer processes. These proteins cycle between ferric and ferrous states [14]. Rubredoxins of different organisms have different redox potentials ranging from -100 to $+50 \mathrm{mV}$. Depending on redox potential, rubredoxins are divided into low potential and high potential groups. To study the redox potential of Mtb RubB, we evaluated the direct electron transfer process on an electrode surface as non-catalytic cycling between oxidized ferric and reduced ferrous states. A solution of RubB in the supporting electrolyte gave no cyclic voltammetric response at bare screen-printed electrode (SPE). However, modification of the SPE with multiwalled carbon nanotubes (MWCNTs) led to well- defined cyclic voltammograms (Fig.2A).

Direct electrochemistry of RubB was achieved by noncovalent immobilization on an MWCNTmodified SPE. Two peaks of the iron-sulfur cluster characterizes reduction and oxidation of RubB. Analysis of voltammograms revealed behavior consistent with quasi-reversibility; indeed, with increased scan rate there was an increase in separation between anodic and cathodic peaks (Fig. 2B). The observed linear dependence of anodic and cathodic peak current on the scan rate indicated that the electron transfer from and to the electrode is a reversible and surface-controlled process. The calculated value of the electrochemical potential of RubB suggest that electron transfer from Mtb reductase FprA is possible (Table 2). Our results are in accordance with previously reported data on electrochemistry of rubredoxins obtained using gold disk or carbon electrodes modified by means of neomycin and $\mathrm{Mg}^{2+}$ ions [15].

\section{Reconstitution of CYP activity}

Mtb CYPs, like most bacterial CYPs, belong to the class I electron-transfer system and are driven by ferredoxin and ferredoxin reductases that transfer the electrons from the NAD $(\mathrm{P}) \mathrm{H}$ to the CYP heme cofactor. Recently, it was shown that activity of several Mtb CYPs could be supported by 
specific cognate redox partners in vitro [16], but redox partners are still unknown for most Mtb CYPs.

It was shown that RubB is co-expressed with CYP125 in a macrophage infection model [17] and with CYP142 during the transition to dormancy [18]; these data link RubB to cholesterol metabolism. To evaluate the ability of RubB to support enzymatic activity of CYPs involved in cholesterol metabolism, we selected CYP124, CYP125, and CYP142 as these are enzymes with well-established catalytic profiles and crystal structures are available [19]. The enzymatic reaction was performed a reconstituted system, containing reductase, RubB, and CYP. Analysis of high performance liquid chromatography (HPLC) profiles clearly demonstrate the formation of the product for all tested CYPs, indicating that recombinant RubB transferred electrons from the reductase to CYP. The amounts of products formed in the presence of RubB were comparable to that produced when the most efficient system with spinach ferredoxin was used [20]. Products were formed with significantly different efficiencies when reductases from various sources were used (Table 3), demonstrating the selectivity of RubB. The most efficient redox-partners for RubB in CYP124-catalyzed reaction were reductases Arh1 from Saccharomyces cerevisiae and PETH from Spinacia oleracea, whereas cognate reductases NADH- ferredoxin reductase FdR and NADPH-ferredoxin reductase FprA resulted in considerably less product. In contrast, for CYP125 the preferred reductase was FprA. Thus, Rub efficiently mediates the hydroxylation reactions of cholesterol-metabolizing CYPs and the product conversion rates were modulated by the reductase component of the system.

\section{Crystal structure of RubB}

The crystal structure of RubB was determined with anisotropic resolution $(1.2 \times 1.3 \times 1.6 \AA)$. The crystals belong to the $\mathrm{P} 1$ space group with eight molecules in the asymmetric unit. All amino acids were built except C-terminal residues A58-S60 of three monomers (in chains B, D and H). Protein molecules in the asymmetric unit did not have significant structural differences with the C $\alpha$ root mean square deviation (RMSD) not exceeding $0.7 \AA$ (Fig. 3).

RubB has a rubredoxin fold similar to the fold previously characterized [21]. In particular, the RubB fold consists of one antiparallel $\beta$-sheet with three strands, which are connected by two loops. Each loop contains a conserved cysteine motif (CXXCG). Cysteines from the loops (Cys9, Cys12, Cys42, and Cys45) surround an iron ion in tetrahedral geometry. This $\mathrm{FeS}_{4}$ cluster is the active site of the protein. Average Fe-S bond lengths are similar to the typical value of $2.3 \AA$ ( 2.33 $\AA, 2.31 \AA, 2.33 \AA, 2.30 \AA$ with $0.03 \AA$ standard deviation between 8 protein copies), indicating the iron is in the oxidized state [21].

Previously, a zinc-substituted RubB structure was solved by NMR (PDB ID: 2KN9). Pairwise C $\alpha$ RMSD values between molecules in our X-ray structure and the NMR-based model do not exceed 1.1 A. In the NMR model, residues at $\mathrm{C}$ and $\mathrm{N}$ termini show backbone variability, while the rest of the structure remain almost unperturbed. There are two major differences in the active sites between the two structures. The first is a rotameric state of D44. The second is that the bond lengths in $\mathrm{FeS}_{4}$ cluster are significantly longer in the NMR model (2.41 $\AA, 2.41 \AA, 2.42 \AA, 2.41 \AA$ ). The 
latter might result from the zinc substitution, as was previously observed for other rubredoxins by different techniques [22, 23].

\section{Role of zinc in structure and function of RubB}

In the crystal structure, we observed a number of intermolecular and intramolecular contacts with zinc ions (Fig. S4) that may be a consequence of Glu and Asp on the surface. Coordination spheres in these contacts are formed by various protein and solvent groups, including carboxyl groups of Glu, Asp, acetate ions, and the C-terminal Ser, carbonyl and amino groups of N-terminal Met, and glycerol.

To determine whether zinc ions influence protein activity or stability we performed CD, ITC, and DSC studies. The CD spectrum of mycobacterial RubB is characterized by a double minimum at 204 and $227 \mathrm{~nm}$ (Fig. S3). The spectrum indicates that protein has not only canonical $\beta$-strands and helices, but also unstructured regions; this is in correspondence with the crystal structure. Addition of zinc did not induce changes in the CD spectrum. Zinc binding was further studied using ITC. The following parameters were estimated: $\mathrm{K}_{\mathrm{d}}=12.0 \pm 0.7 \mu \mathrm{M}, \Delta \mathrm{H}=30.4 \pm 0.9 \mathrm{kcal} / \mathrm{mol}$, $-\mathrm{T} \Delta \mathrm{S}=-36.9 \pm 1 \mathrm{kcal} / \mathrm{mol}$, and $\Delta \mathrm{G}=-6.5 \pm 0.3 \mathrm{kcal} / \mathrm{mol}$ (Fig. S5). The obtained data demonstrated that zinc binds to RubB with high affinity and that binding is entropy-driven and thermodynamically favorable.

Next, we measured the activity of CYP124 in the presence of increasing zinc concentrations with another doubly charged metal ion $\left(\mathrm{Ni}^{2+}\right)$ as reference (Table 4). A dramatic decrease of activity was observed as the zinc concentration was increased with almost no activity observed at the 100 $\mu \mathrm{M} \mathrm{Zn}{ }^{2+}$ in a reaction mixture with CYP124, RubB, and Arh1 reductase. In comparison, a $10 \mathrm{mM}$ $\mathrm{Ni}^{2+}$ concentration decreased activity only 2 fold compared to activity in the absence of added ion. These experiments do not allow us to conclude which components of the redox system are affected by the presence of zinc. The interaction between redox-partners and/or direct intersection with the catalytic cycle of CYP may be inhibited by the specific metal "poisoning" effect.

Since zinc ions participate in crystal contacts (Fig. S4), we hypothesized that RubB might become disfunction because of oligomerization in the presence of high concentrations of $\mathrm{Zn}^{2+}$. We performed size-exclusion chromatography-multiple angle light scattering analysis of RubB in the presence and absence of $\mathrm{Zn}^{2+}$. In both the presence and absence of $\mathrm{Zn}^{2+}$, RubB a homogeneous peak was observed with a mass of $6.6 \pm 0.3 \mathrm{kDa}$, corresponding to monomer. Thus, RubB does not appear to form dimers or oligomers in the presence of zinc. We further analyzed thermal stability of RubB in presence of increasing $\mathrm{Zn}^{2+}$ concentrations (Table 5). Only at high concentrations (10 $\mathrm{mM} \mathrm{Zn}^{2+}$ ) were two unfolding events detected. The observed decrease in stability might be explained by the partial iron substitution of zinc for iron that occurs during heating.

\section{Isothermal calorimetry analysis of the interaction between RubB and CYP124}

To characterize complex formation between CYP124 and RubB from a thermodynamic point of view, entropy, enthalpy, and Gibbs energy values were estimated by the ITC. RubB binds to CYP124 with dissociation constant of $12.8 \mu \mathrm{M}$. Complex formation is thermodynamically 
favorable and entropy and enthalpy driven (Table 6). To probe if the substrate binding induces conformational changes in CYP124 that influence binding of RubB, we performed ITC measurements in the presence of the CYP124 substrate cholestenone. Addition of cholestenone decreased affinity of CYP124 for RubB (Table 6), indicating that RubB preferentially binds to the ligand-free CYP124. The same effect was observed upon the interaction between electron transfer proteins P450cam and Pdx in Pseudomonas putida [24]. A decrease in affinity of CYP124 for RubB was also observed when the ionic strength of a solution was increased, indicating that electrostatic interactions stabilize the complex.

\section{Probing protein-protein interactions by site-directed mutagenesis}

In order to identify specific amino acids of RubB important for the activity, we first performed multiple sequence alignment (Fig. S6). The residues Q11, W22, S41, and D44 were outliers. It was previously proposed that L41 in Clostridium pasteurianum rubredoxin, which corresponds to D44 in RubB, acts as a charge-dependent gate to control solvent access to the FeS cluster and facilitate electron transfer [25]. Moreover, D44 conformations are different in our crystal structure than in the reported NMR structure (Fig. S7), suggestive of the functional relevance of this residue. We prepared RubB mutants in which residues were changed to those in other rubredoxins $(\mathrm{Q} 11 \mathrm{~V}$, W22D, S41V, and D44A) and in which charge-neutralizing changes were made (E24A, D25A, D34A, D35A, D38A, and D39A). None of these mutants had characteristics different from the wild type RubB during expression and purification.

To assess whether mutations affected the structure of RubB, we performed CD. Comparison of spectra of wild type and mutant RubB indicated that mutations did not induce significant structural perturbations. We next examined effects of the mutations on thermal stability of RubB (Fig. S8). In all cases, DSC data fit a two-state model within the expected error of data collection. E24A and D25A mutations did not have an effect on RubB thermal stability. D44A, S41V, Q11V mutations increased the thermal transition temperature $\left(+5.5^{\circ} \mathrm{C},+1.7{ }^{\circ} \mathrm{C},+2.4{ }^{\circ} \mathrm{C}\right.$ relative to wild type, respectively). The profound effect of the $\mathrm{D} 44 \mathrm{~A}$ mutation on thermal stability indicates that charge neutralization in this position increases overall stability of RubB (Table 7).

We next measured catalytic activity of CYP124 in the presence of RubB mutants in reconstituted system containing Arh1, FprA, and FdR (Fig. 4). A 2-fold difference between the wild type and mutant was considered a significant effect. When the Mtb reductase FdR was used, no significant decrease in enzymatic activity of CYP124 was detected for any RubB mutant relative to that in the presence of wild type RubB. In the case of Mtb reductase FprA and the heterologous redox protein Arh1, the D44A mutation resulted in a decrease of CYP124 activity by 2.2-fold, whereas other mutations had no effect. It is possible that D44 of RubB interacts with the reductase. To test this, we superimposed our crystal structure of RubB with that of the complex of rubredoxin and rubredoxin reductase from Pseudomonas aeruginosa [PDB ID: 2V3B]. The residues E24 and D44 of RubB correspond to E21 and D41 of rubredoxin from $P$. aeruginosa. These two residues form salt bridges with the reductase [26]. The rubredoxin-rubredoxin reductase complex has high charge complementarity yet is transient. This comparison suggests that effects of corresponding mutation 
at D44 of RubB could be explained by the impaired interactions with solvent exposed residues of FprA or Arh1 reductases.

We then compared the effects of RubB mutations on catalytic activity of CYP124, CYP125, and CYP142. We chose the cognate reductase FprA as a redox-partner, because catalytic activities of CYP125 and CYP142 was low in the reconstituted system containing FdR. The Q11V mutation, which introduces a residue conserved in other rubredoxins (Fig. S6), slightly increased catalytic activities of CYP124, CYP125, and CYP142. Similarly, D35 and D39 mutations led to an increase in CYP125 activity of about 1.5 fold but did not alter activities of CYP142 and CYP124 (Fig. 5). In the presence of the RubB D25A mutant, there was a 6.4-fold decrease in catalytic activity of CYP142 compared to activity in the presence of the wild type RubB, but CYP125 and CYP124 activities were unchanged (Fig. 5). The proximal surface of CYP142 is less positively charged than those of CYP124 and CYP125 (Fig. 6), suggesting that RubB D25A mutation affects the electrostatic complementarity between RubB and CYP142.

Mutations E24A, D44A, and D38A in RubB led to decreases in enzymatic activity of CYP125 and CYP142, with D44A the most influential reducing catalysis of all three CYPs (Fig. 5). Similar mutation of aspartate residue near iron-sulfur cluster was previously studied in the putidaredoxin from $P$. putida where D38A mutation resulted in almost complete loss of catalytic activity of CYP101A1 [27]. The effect of the D44A mutation was not as dramatic, suggesting rather low selectivity for RubB. These data support our hypothesis that D44 is involved in the interaction with reductase as sites of RubB interaction with both reductase and CYPs could be the same. Considering the small size of RubB it is reasonable to assume that it functions as a shuttle within CYP-dependent systems. RubB is very tolerant to amino acid substitutions as single charge neutralizations did not dramatically affect the CYP-RubB interactions. The RubB thus probably forms transient non-specific complexes within the CYP-dependent system.

\section{Discussion}

Rubredoxins facilitate redox reactions and electron transfer as a single protein or as a domain within different protein families. Their functions vary from facilitation of adaptation to a changing redox environment [28] to the maintenance of overall protein stability [29] and are associated with different developmental processes [30]. In present study, we demonstrated that the Mtb rubredoxin RubB is a potent redox partner for Mtb CYPs. CYPs catalyze stereo- and regioselective oxidation of various endogenous substrates and produce bioactive compounds essential for medicine, agriculture and industry; they are indispensable components of biotechnological production of expensive or complex molecules [31]. The function of many CYPs of Mtb are unknown. The ability of RubB to transfer electrons from different reductases to at least three CYPs will make RubB an important research tool in the cytochrome P450 field.

Chemically modified electrodes such as those modified with carbon nanomaterials are used for the investigation of direct electrochemistry of metalloproteins. Composites based on carbon nanomaterials are promising for electrode modification to improve analytical parameters and sensor performance [32-34]. Based on our experimental data on electrochemistry of RubB carbon 
nanotubes are suitable promoters for negatively charged rubredoxin at carbon screen-printed electrodes. Carbon nanostructures possess electrochemically active surfaces and metallic or semiconductor properties that can induce catalysis by participating in electron transfer processes $[35,36]$. An external supply of electrons using such electrodes via rubredoxin to CYPs may represent a new electron source for biomedical and biotechnological applications.

CYP124 catalyzes selective vitamin D3 hydroxylation, producing vitamin D3 metabolites with high biotechnological potential. An important focus of engineering of P450-dependent pathways is reducing equivalents to drive the catalytic cycle. The small size and high stability of RubB could be used in design of light-driven fusion proteins suitable for biotechnological applications, similarly to the fusion of ferredoxin and plant CYP79A1, which receives electrons directly from photosystem I [37]. Even more exciting from our point of view is the possibility of design of "infusion" CYPs. The rubredoxin fold retains electron transfer properties even in the context of an 18- amino acid peptide [38]. It may be possible to insert such a miniaturized rubredoxin into the meander region of CYP on the proximal surface (where the redox partner binds) to obtain infusion proteins suitable for a wide variety of applications.

Rubredoxin could also be used as a tag for screening of expression of colorless proteins [39], or to increase yields during expression of highly basic proteins.

Our finding that RubB is able to support catalytic activity of cholesterol-metabolizing CYPs furthers our understanding of these Mtb proteins. CYPs are essential for bacterial viability and pathogenicity [40]. A rubredoxin domain in the Mtb protein kinase $\mathrm{G}$ is important for kinase function under different redox conditions [28]. We suggest that rubredoxin motifs in Mtb proteins enable the bacterium to adapt to different microenvironments during its life cycle. In case of CYPs, RubB may participate in a switch mechanism from classic ferredoxins under iron starvation.

In summary, our studies revealed that RubB supports catalytic activity of heme-containing proteins of cytochrome P450 family in Mtb. From a broad perspective, our result have value in the biotechnology and synthetic biology applications of cytochrome P450 catalysis.

\section{Materials and methods}

\section{Cloning and expression of Mtb RubB}

The $R u b B(\mathrm{Rv} 3250 \mathrm{c})$ gene was amplified from Mtb genomic DNA. The amplification reaction product was ligated into the expression vector pET11a.

E. coli $\mathrm{C} 43$ competent cells were transformed with pET11a containing RubB. Transformed cells were screened on Petri dishes with LB-agar containing ampicillin $(100 \mu \mathrm{g} / \mathrm{ml})$. An overnight culture $(3 \mathrm{ml})$ was used to inoculate $0.5 \mathrm{~L}$ of TB-medium containing $100 \mathrm{mM}$ potassium-phosphate buffer, $\mathrm{pH} 7.4$, and $100 \mu \mathrm{g} / \mathrm{ml}$ ampicillin. The mixture was incubated in a thermostated orbital shaker $(180 \mathrm{rpm})$ at $37^{\circ} \mathrm{C}$. After absorbance at $600 \mathrm{~nm}$ reached $\sim 0.4$, RubB expression was induced by IPTG (final concentration $0.5 \mathrm{mM}$ ), additional $\mathrm{FeCl}_{3}$ (final concentration $100 \mu \mathrm{g} / \mathrm{ml}$ ). After $20 \mathrm{~h}$ of incubation at $20^{\circ} \mathrm{C}$ with shaking at $100 \mathrm{rpm}$, the cells were collected by centrifugation (8000 g, $10 \mathrm{~min}$ ). The pellet was resuspended in $50 \mathrm{mM}$ potassium phosphate buffer, $\mathrm{pH} 7.4$, containing $20 \%$ glycerol, $0.1 \mathrm{mM}$ EDTA, and $0.5 \mathrm{mM}$ PMSF. The cells were stored at $-73{ }^{\circ} \mathrm{C}$. 


\section{Purification of RubB}

The cell suspension was sonicated in an ice-water bath (7 x 1-min pulses with 1-min intervals). The suspension was centrifuged for $1 \mathrm{~h}$ at 20,500 rpm and the supernatant was applied to a DEAESepharose column equilibrated with buffer A (50 mM potassium phosphate buffer, pH 7.4, containing 0.1 mM EDTA). The column was washed with 2-3 volumes of buffer A, and then with 10 volumes of buffer A containing $15 \mathrm{mM} \mathrm{NaCl}$. RubB was eluted from the column with buffer A containing $200 \mathrm{mM} \mathrm{NaCl}$. Eluted fractions were applied to a Superdex 75 16/60 column equilibrated with buffer A containing $200 \mathrm{mM} \mathrm{NaCl}$. The colored band containing RubB was collected.

\section{Analytical methods}

RubB purity was shown to be $>95 \%$ by SDS-PAGE. Spectral studies were performed on a Cary200 spectrophotometer in $50 \mathrm{mM}$ potassium phosphate buffer, $\mathrm{pH}$ 7.4. The concentration of RubB was determined using molar absorption coefficient of $6.9 \mathrm{mM}^{-1} \mathrm{~cm}^{-1}$ at $490 \mathrm{~nm}$ [41].

\section{Electrochemical measurements}

Electrochemical measurements were carried out using an EmStat3 potentiostat (PalmSens) equipped with the PSTrace software (version 5.4). Three-pronged electrodes obtained by screenprinting with graphite were used as working and auxiliary electrodes, and a silver chloride electrode was used as the reference electrode (http://www.colorel.ru). The diameter of the working electrode was $0.2 \mathrm{~cm}$ (area $0.0314 \mathrm{~cm}^{2}$ ). Electrochemical measurements were performed at room temperature in $0.1 \mathrm{M}$ potassium phosphate buffer $(\mathrm{pH} 7.4)$, containing $0.05 \mathrm{M} \mathrm{NaCl}$. Cyclic voltammograms were obtained at a scan rate of $0.05-0.1 \mathrm{~V} \mathrm{~s}^{-1}$ and potential range from +0.5 to $0.8 \mathrm{~V}$ (vs. $\mathrm{Ag} / \mathrm{AgCl}$ ). All potentials are referred to relative to the $\mathrm{Ag} / \mathrm{AgCl}$ reference electrode. The average values from three independent experiments are reported.

Prior to measurements, the SPEs were modified by aqueous dispersions of MWCNTs (SigmaAldrich) obtained from solubilized by amphiphilic poly(1,2-butadiene)-block-poly(2-( N,Ndimethylamino)ethyl methacrylate) ( $\mathrm{PB}_{290}-b$-PDMAEMA $\left.{ }_{240}\right)$ diblock copolymer for a mean diameter of $9.5 \mathrm{~nm}$ and a length of $1 \mu \mathrm{m}$. A detailed description of the polymer synthesis, dispersion preparation, and the physico-chemical features of the modified SPEs can be found elsewhere [34], [42]. For preparation of the modified electrodes, $2 \mu \mathrm{L}$ of the aqueous $\mathrm{PB}_{290}-b$ PDMAEMA $240 /$ MWCNT dispersion was dropped onto an area of the SPE and incubated for 15 min at $37^{\circ} \mathrm{C}$ until completely dry [33, 34].

For further incorporation of RubB, $1 \mu \mathrm{L}$ of a solution of RubB at an appropriate concentration was applied to the surface of the modified SPE. The electrodes were allowed to stand for $12 \mathrm{~h}$ at $4{ }^{\circ} \mathrm{C}$ in a humid chamber. During the investigation of RubB potential, electrodes were in a planar regimen and covered by $60 \mu \mathrm{L}$ of $0.1 \mathrm{M}$ potassium phosphate buffer $(\mathrm{pH} 7.4)$, containing $0.05 \mathrm{M}$ $\mathrm{NaCl}$. Typical peak current separation $\left(\triangle E_{p}\right)$ at $100 \mathrm{mV} \mathrm{s}^{-1}$ scan rate was $183 \mathrm{mV}$. The immobilized RubB showed two peaks, the reduction peak (the cathodic $E_{\mathrm{pc}}$ ) and the oxidation peak (the anodic $E_{\text {pa }}$ ), in direct non-catalytic cyclic voltammetry with midpoint potentials from $-231 \mathrm{mV}$ to -264 
$\mathrm{mV}$. The midpoint potential $E^{01}$ was calculated from the values of anodic and cathodic peak potentials using the equation $E^{01}=\left(E_{\mathrm{pc}}+E_{\mathrm{pa}}\right) / 2$.

\section{Circular dichroism spectroscopy}

A calibrated Jasco Model J-820 spectropolarimeter was used to collect CD data from $0.3 \mathrm{mg} / \mathrm{ml}$ RubB samples in $50 \mathrm{mM}$ potassium-phosphate buffer, $\mathrm{pH}$ 7.4. Spectra were recorded in a quartz cell of 1-cm path length between 200 and $260 \mathrm{~nm}$ at $298 \mathrm{~K}$. Spectra were recorded in triplicate with a bandwidth of $1.0 \mathrm{~nm}$ and a time constant of $1.0 \mathrm{~s}$, and the average was reported. The average spectra were processed by subtracting a blank spectrum from the protein spectrum.

\section{Isothermal titration calorimetry for RubB binding to CYP124}

Experiments were performed using an ITC200 calorimeter equipped with the control and data acquisition and analysis software ORIGIN 7 (MicroCal Inc.). RubB and CYP124 were dialyzed overnight against $50 \mathrm{mM}$ potassium-phosphate buffer, $\mathrm{pH} 7.4$. An aliquot of $20 \mu \mathrm{M}$ CYP124 was placed in the calorimetric cell and titrated with $1 \mathrm{mM}$ RubB. The first injection $(1 \mu 1$, omitted from the analysis) was followed by 20 injections of $4 \mu 1$ at 2 -min intervals. The titration syringe was continuously stirred at $750 \mathrm{rpm}$, and the temperature of the calorimetric cell was $25^{\circ} \mathrm{C}$. Injecting the RubB into the buffer alone was used as reference titration. When CYP124 substrate cholestenone was tested, cholestenone was dissolved in DMSO and was added to CYP124 at the final concentration $5 \mu \mathrm{M}$. DMSO was added to RubB in the same amount.

\section{Isothermal titration calorimetry for metal binding to RubB}

RubB was dialyzed overnight against $50 \mathrm{mM}$ HEPES, pH 7.4. RubB $(0.2 \mathrm{mg} / \mathrm{ml})$ was placed in the calorimetric cell and titrated with $200 \mathrm{mM}$ zinc acetate dissolved in $50 \mathrm{mM}$ HEPES, pH 7.4. The first injection ( $1 \mu 1$, omitted from the analysis) was followed by 20 injections of $4 \mu 1$ at 2 -min intervals. The titration syringe was continuously stirred at $750 \mathrm{rpm}$ and the temperature of the calorimetric cell was $25^{\circ} \mathrm{C}$. Injection of the zinc acetate into the buffer alone was used as a reference titration.

\section{Differential scanning calorimetry studies of RubB}

DSC experiments were performed using a Microcal VP-DSC instrument (Malvern Instruments). The temperature gradient was $20^{\circ} \mathrm{C}$ to $100^{\circ} \mathrm{C}$, the scan rate $60^{\circ} \mathrm{C} / \mathrm{h}$. Baseline scans were of assay buffer (50 mM HEPES, pH 5.0). RubB was diluted to a final concentration of $50 \mu \mathrm{M}$ in the same buffer. Zinc acetate was added to a final concentration of $10 \mathrm{mM}$. For data analysis Origin Software (OriginLab) was used.

\section{Catalytic activity of CYP124, CYP125, CYP142}

Expression and purification of CYP124, CYP125, and CYP142 was performed as described previously [43]. Expression and purification of Arh1 and FprA was performed as described previously [44]. 
Catalytic activity of CYP124, CYP125 and CYP142 was determined in the reconstituted system containing $0.5 \mu \mathrm{M}$ CYP, $1 \mu \mathrm{M}$ FprA and $5 \mu \mathrm{M}$ RubB in $25 \mathrm{mM}$ HEPES, pH 7.4 containing 100 $\mu \mathrm{M}$ of 7-ketocholesterol (for CYP124) or cholestenone (for CYP142 and CYP125). Aliquots of concentrated proteins were mixed and pre-incubated for $5 \mathrm{~min}$ at room temperature. After incubation at $30^{\circ} \mathrm{C}$ for $5 \mathrm{~min}$, the reaction was started by adding NADPH-regeneration system (3.3 $\mathrm{mg} / \mathrm{ml}$ glucose-6-phosphate, $0.16 \mathrm{mg} / \mathrm{ml} \mathrm{NADPH}, 0.6 \mu \mathrm{l} / \mathrm{ml}$ glucose-6-phosphate dehydrogenase). The mixture was incubated at $30{ }^{\circ} \mathrm{C}$, and aliquots $(0.5 \mathrm{ml})$ were taken at 15 and $30 \mathrm{~min}$ time intervals. Reactions were stopped by addition of $5 \mathrm{ml}$ of methylene chloride, vortexed, and centrifuged at 3,000 rpm for $5 \mathrm{~min}$. The organic layer was dried under argon flow, $100 \mu \mathrm{l}$ of methanol was added to the pellet, and products were analyzed by HPLC using a C18 Luna $100 \AA$, $250 \times 4.6 \mathrm{~mm}$ column on an Agilent Technologies 1200 Series instrument.

\section{Crystallization, data collection, X-ray crystallography, and modeling of Rub-CYP complex} Purified RubB was diluted to $1.5 \mathrm{mM}$ in $10 \mathrm{mM}$ Tris-HCl, $\mathrm{pH} 7.4$ for crystallization trials. The initial screening was performed using commercially available screening kits from Qiagen and Molecular Dimensions in 96-well plate format using sitting-drop technique. Red-colored crystals of RubB grew in 2 days from $0.05 \mathrm{M}$ zinc acetate, 20\% PEG3350 at $20{ }^{\circ} \mathrm{C}$. Optimization of crystallization conditions was carried out manually in 96 -well plates using sitting-drop protocol. The best crystals are grown in $100 \mathrm{mM}$ Tris- $\mathrm{HCl} \mathrm{pH}$ 7.5, $50 \mathrm{mM}$ zinc acetate, 25\% PEG3350.

Diffraction data were collected at the European Synchrotron Radiation Facility (ESRF) beamline ID30B. The data collection strategy was optimized in BEST [45]. All data were processed in the XDS software package [46]. Processed data were corrected for anisotropy using the STARANISO server [http://staraniso.globalphasing.org/cgi-bin/staraniso.cgi]. A local mean $I / \sigma(I)$ value of 1.20 was used to determine the anisotropic diffraction-limit surface.

The phase problem was solved in the automatic molecular replacement pipeline MoRDa [47]. The obtained space group was P1 with eight molecules per asymmetric unit. The model was rebuilt in phenix.autobuild [48]. Multiple rounds of model refinement with anisotropic B-factors were done in refmac5 [49] and phenix.refine [50]. Manual refinement was performed in Coot [51]. The quality of the resulting model was analyzed using phenix.molprobity [52] and QCCheck (https://smb.slac.stanford.edu/jcsg/QC/). The secondary structure was validated using the DSSP program [53]. Zinc ion geometry was determined using the CheckMyMetal web server [54, 55]. Data collection and final refinement statistics are presented in Table S1.

\section{Site-directed mutagenesis of RubB}

Mutations were introduced by site-directed mutagenesis using oligonucleotides listed in Table S2. PCR conditions for single amino acid mutations were as follows: 25 cycles of $10 \mathrm{~s}$ at $98^{\circ} \mathrm{C}, 5 \mathrm{~s}$ at $60^{\circ} \mathrm{C}$, followed by $6 \mathrm{~min}$ at $68^{\circ} \mathrm{C}$. The resulting mutant plasmids were verified by DNA sequencing.

\section{Size-exclusion chromatography-multiple angle light scattering experiments}


The experimental system consisted of a size-exclusion column and an HPLC equipped with a DAWN MALS detector (Wyatt Technology) and an Optilab differential refractive index detector (Wyatt Technology). RubB diluted in $50 \mathrm{mM}$ HEPES, pH 7.4, containing $0.05 \mathrm{M} \mathrm{NaCl}$ to a final concentration of $1 \mathrm{mg} / \mathrm{ml}$ was applied to a Superdex 10/300 column (GE Healthcare) run at a flow rate of $0.5 \mathrm{ml} / \mathrm{min}$. Zinc chloride was added to a final concentration of $5 \mathrm{mM}$ if needed. Radius of gyration and absolute molecular mass were calculated using Wyatt's ASTRA software.

\section{Author contributions}

Conceptualization, A.G., N.S.;

Methodology, V.B., V.S., K.T., A.G., N.S.;

Investigation, T.S., A.K., I.G., An.K., D.V., S.B., E.M., Al.K., R.M., L.S., N.S.;

Writing - Original Draft. T.S., D.V., N.S.;

Writing - Review \& Editing, S.B., V.B., A.G., N.S.;

Funding Acquisition, V.B., A.G., N.S.;

Supervision, V.B., A.G., N.S.

\section{Acknowledgments}

This study was inspired by personal communication with Prof. Trevor Forsyth (The Institut LaueLangevin, France). This work was supported by a joint grant received from Belarusian Republican Foundation for Fundamental Research, B20R-061 and Russian Foundation for Basic Research, 20-54-00005. V.B. is supported by the Ministry of Science and Higher Education of the Russian Federation (agreement \#075-00337-20-03, project FSMG-2020-0003). We thank Dr. Dmitry V. Pergushov and Dr. Felix H. Schacher for their help with electrode modifiers. The electrode modifiers were developed in the frames of Russian Science Foundation (RSF, project no. 18-4404011) and the Deutsche Forschungsgemeinschaft (DFG, SCHA1640/18-1) within a joint RSFDFG grant. We acknowledge the ESRF Structural Biology Group. 


\section{References}

1. Buchmeier, N. A., Newton, G. L. \& Fahey, R. C. (2006) A mycothiol synthase mutant of Mycobacterium tuberculosis has an altered thiol-disulfide content and limited tolerance to stress, Journal of bacteriology. 188, 6245-52.

2. Ng, V. H., Cox, J. S., Sousa, A. O., MacMicking, J. D. \& McKinney, J. D. (2004) Role of KatG catalase-peroxidase in mycobacterial pathogenesis: countering the phagocyte oxidative burst, Molecular microbiology. 52, 1291-302.

3. Lama, A., Pawaria, S., Bidon-Chanal, A., Anand, A., Gelpi, J. L., Arya, S., Marti, M., Estrin, D. A., Luque, F. J. \& Dikshit, K. L. (2009) Role of Pre-A motif in nitric oxide scavenging by truncated hemoglobin, HbN, of Mycobacterium tuberculosis, The Journal of biological chemistry. 284, 14457-68.

4. Kurthkoti, K., Amin, H., Marakalala, M. J., Ghanny, S., Subbian, S., Sakatos, A., Livny, J., Fortune, S. M., Berney, M. \& Rodriguez, G. M. (2017) The Capacity of Mycobacterium tuberculosis To Survive Iron Starvation Might Enable It To Persist in Iron-Deprived Microenvironments of Human Granulomas, mBio. 8.

5. von Heijne, G. \& Blomberg, C. (1978) Early evolution of cellular electron transport: molecular models for the ferredoxin-rubredoxin-flavodoxin region, Origins of life. 9, 27-37.

6. Boshoff, H. I., Myers, T. G., Copp, B. R., McNeil, M. R., Wilson, M. A. \& Barry, C. E., 3rd (2004) The transcriptional responses of Mycobacterium tuberculosis to inhibitors of metabolism: novel insights into drug mechanisms of action, The Journal of biological chemistry. 279, 4017484.

7. Lin, W., de Sessions, P. F., Teoh, G. H., Mohamed, A. N., Zhu, Y. O., Koh, V. H., Ang, M. L., Dedon, P. C., Hibberd, M. L. \& Alonso, S. (2016) Transcriptional Profiling of Mycobacterium tuberculosis Exposed to In Vitro Lysosomal Stress, Infection and immunity. 84, 2505-23.

8. Coulter, E. D. \& Kurtz, D. M., Jr. (2001) A role for rubredoxin in oxidative stress protection in Desulfovibrio vulgaris: catalytic electron transfer to rubrerythrin and two-iron superoxide reductase, Archives of biochemistry and biophysics. 394, 76-86.

9. Vandal, O. H., Nathan, C. F. \& Ehrt, S. (2009) Acid resistance in Mycobacterium tuberculosis, Journal of bacteriology. 191, 4714-21.

10. Buchko, G. W., Hewitt, S. N., Napuli, A. J., Van Voorhis, W. C. \& Myler, P. J. (2011) Solution-state NMR structure and biophysical characterization of zinc-substituted rubredoxin B (Rv3250c) from Mycobacterium tuberculosis, Acta crystallographica. 67, 1148-53.

11. Prince, C. \& Jia, Z. (2015) An Unexpected Duo: Rubredoxin Binds Nine TPR Motifs to Form LapB, an Essential Regulator of Lipopolysaccharide Synthesis, Structure. 23, 1500-1506.

12. Prakash, S., Sundd, M. \& Guptasarma, P. (2014) The key to the extraordinary thermal stability of P. furiosus holo-rubredoxin: iron binding-guided packing of a core aromatic cluster responsible for high kinetic stability of the native structure, PloS one. 9, e89703.

13. Bonomi, F., Fessas, D., Iametti, S., Kurtz, D. M., Jr. \& Mazzini, S. (2000) Thermal stability of Clostridium pasteurianum rubredoxin: deconvoluting the contributions of the metal site and the protein, Protein Sci. 9, 2413-26.

14. Swartz, P. D., Beck, B. W. \& Ichiye, T. (1996) Structural origins of redox potentials in Fe-S proteins: electrostatic potentials of crystal structures, Biophysical journal. 71, 2958-69.

15. Correia dos Santos, M. M., Paes de Sousa, P. M., Simões Gonçalves, M. L., Ascenso, C., Moura, I. \& Moura, J. J. G. (2001) Electrochemical studies of rubredoxin from Desulfovibrio vulgaris at modified electrodes, J Electroanal Chem. 501, 173-179. 
16. Ortega Ugalde, S., de Koning, C. P., Wallraven, K., Bruyneel, B., Vermeulen, N. P. E., Grossmann, T. N., Bitter, W., Commandeur, J. N. M. \& Vos, J. C. (2018) Linking cytochrome P450 enzymes from Mycobacterium tuberculosis to their cognate ferredoxin partners, Applied microbiology and biotechnology. 102, 9231-9242.

17. Rohde, K. H., Veiga, D. F., Caldwell, S., Balazsi, G. \& Russell, D. G. (2012) Linking the transcriptional profiles and the physiological states of Mycobacterium tuberculosis during an extended intracellular infection, PLoS pathogens. 8, e1002769.

18. Ignatov, D. V., Salina, E. G., Fursov, M. V., Skvortsov, T. A., Azhikina, T. L. \& Kaprelyants, A. S. (2015) Dormant non-culturable Mycobacterium tuberculosis retains stable low-abundant mRNA, BMC genomics. 16, 954.

19. Hudson, S. A., McLean, K. J., Munro, A. W. \& Abell, C. (2012) Mycobacterium tuberculosis cytochrome P450 enzymes: a cohort of novel TB drug targets, Biochemical Society transactions. 40, 573-9.

20. Varaksa, T., Bukhdruker, S., Grabovec, I., Marin, E., Kavaleuski, A., Gusach, A., Kovalev, K., Maslov, I., Luginina, A., Zabelskiy, D., Astashkin, R., Shevtsov, M., Smolskaya, S., Kavaleuskaya, A., Shabunya, P., Baranovsky, A., Dolgopalets, V., Charnou, Y., Savachka, A., Litvinovskaya, R., Hurski, A., Shevchenko, E., Rogachev, A., Mishin, A., Gordeliy, V., Gabrielian, A., Hurt, D. E., Nikonenko, B., Majorov, K., Apt, A., Rosenthal, A., Gilep, A., Borshchevskiy, V. \& Strushkevich, N. (2020) Metabolic fate of human immunoactive sterols in $<$ em $>$ Mycobacterium tuberculosis $</$ em $>$, bioRxiv, 2020.07.07.192294.

21. Liu, J., Chakraborty, S., Hosseinzadeh, P., Yu, Y., Tian, S., Petrik, I., Bhagi, A. \& Lu, Y. (2014) Metalloproteins containing cytochrome, iron-sulfur, or copper redox centers, Chemical reviews. 114, 4366-469.

22. Dauter, Z., Wilson, K. S., Sieker, L. C., Moulis, J. M. \& Meyer, J. (1996) Zinc- and ironrubredoxins from Clostridium pasteurianum at atomic resolution: a high-precision model of a $\mathrm{ZnS} 4$ coordination unit in a protein, Proceedings of the National Academy of Sciences of the United States of America. 93, 8836-40.

23. Guo, Y., Brecht, E., Aznavour, K., Nix, J. C., Xiao, Y., Wang, H., George, S. J., Bau, R., Keable, S., Peters, J. W., Adams, M. W., Jenney, F., Sturhahn, W., Alp, E. E., Zhao, J., Yoda, Y. \& Cramer, S. P. (2013) Nuclear resonance vibrational spectroscopy (NRVS) of rubredoxin and MoFe protein crystals, Hyperfine interactions. 222, 77-90.

24. Hollingsworth, S. A., Batabyal, D., Nguyen, B. D. \& Poulos, T. L. (2016) Conformational selectivity in cytochrome P450 redox partner interactions, Proceedings of the National Academy of Sciences of the United States of America. 113, 8723-8.

25. Park, I. Y., Youn, B., Harley, J. L., Eidsness, M. K., Smith, E., Ichiye, T. \& Kang, C. (2004) The unique hydrogen bonded water in the reduced form of Clostridium pasteurianum rubredoxin and its possible role in electron transfer, J Biol Inorg Chem. 9, 423-8.

26. Hagelueken, G., Wiehlmann, L., Adams, T. M., Kolmar, H., Heinz, D. W., Tummler, B. \& Schubert, W. D. (2007) Crystal structure of the electron transfer complex rubredoxin rubredoxin reductase of Pseudomonas aeruginosa, Proceedings of the National Academy of Sciences of the United States of America. 104, 12276-81.

27. Kuznetsov, V. Y., Poulos, T. L. \& Sevrioukova, I. F. (2006) Putidaredoxin-to-cytochrome P450cam electron transfer: differences between the two reductive steps required for catalysis, Biochemistry. 45, 11934-44.

28. Wittwer, M., Luo, Q., Kaila, V. R. \& Dames, S. A. (2016) Oxidative Unfolding of the Rubredoxin Domain and the Natively Disordered N-terminal Region Regulate the Catalytic 
Activity of Mycobacterium tuberculosis Protein Kinase G, The Journal of biological chemistry. 291, 27062-27072.

29. Garcia-Cerdan, J. G., Furst, A. L., McDonald, K. L., Schunemann, D., Francis, M. B. \& Niyogi, K. K. (2019) A thylakoid membrane-bound and redox-active rubredoxin (RBD1) functions in de novo assembly and repair of photosystem II, Proceedings of the National Academy of Sciences of the United States of America. 116, 16631-16640.

30. Li, Y., Liu, P. P. \& Ni, X. (2019) Molecular Evolution and Functional Analysis of RubredoxinLike Proteins in Plants, Biomed Res Int. 2019, 2932585.

31. Putkaradze, N., Konig, L., Kattner, L., Hutter, M. C. \& Bernhardt, R. (2020) Highly regioand stereoselective hydroxylation of vitamin D2 by CYP109E1, Biochemical and biophysical research communications. 524, 295-300.

32. Carrara, S., Baj-Rossi, C., Boero, C. \& De Micheli, G. (2014) Do Carbon Nanotubes contribute to Electrochemical Biosensing?, Electrochimica Acta. 128, 102-112.

33. Shumyantseva, V. V., Sigolaeva, L. V., Agafonova, L. E., Bulko, T. V., Pergushov, D. V., Schacher, F. H. \& Archakov, A. I. (2015) Facilitated biosensing via direct electron transfer of myoglobin integrated into diblock copolymer/multi-walled carbon nanotube nanocomposites, $J$ Mater Chem B. 3, 5467-5477.

34. Shumyantseva, V. V., Bulko, T. V., Kuzikov, A. V., Masamrekh, R. A., Konyakhina, A. Y., Romanenko, I., Max, J. B., Kohler, M., Gilep, A. A., Usanov, S. A., Pergushov, D. V., Schacher, F. H. \& Sigolaeva, L. V. (2020) All-electrochemical nanocomposite two-electrode setup for quantification of drugs and study of their electrocatalytical conversion by cytochromes P450, Electrochimica Acta. 336, 135579.

35. Justino, C. I. L., Rocha-Santos, T. A. P. \& Duarte, A. C. (2013) Advances in point-of-care technologies with biosensors based on carbon nanotubes, Trac-Trend Anal Chem. 45, 24-36.

36. Arduini, F., Micheli, L., Moscone, D., Palleschi, G., Piermarini, S., Ricci, F. \& Volpe, G. (2016) Electrochemical biosensors based on nanomodified screen-printed electrodes: Recent applications in clinical analysis, TrAC Trends in Analytical Chemistry. 79, 114-126.

37. Mellor, S. B., Nielsen, A. Z., Burow, M., Motawia, M. S., Jakubauskas, D., Moller, B. L. \& Jensen, P. E. (2016) Fusion of Ferredoxin and Cytochrome P450 Enables Direct Light-Driven Biosynthesis, ACS chemical biology. 11, 1862-9.

38. Jacques, A., Clemancey, M., Blondin, G., Fourmond, V., Latour, J. M. \& Seneque, O. (2013) A cyclic peptide-based redox-active model of rubredoxin, Chemical communications (Cambridge, England). 49, 2915-7.

39. Kohli, B. M. \& Ostermeier, C. (2003) A Rubredoxin based system for screening of protein expression conditions and on-line monitoring of the purification process, Protein expression and purification. 28, 362-7.

40. Ortega Ugalde, S., Boot, M., Commandeur, J. N. M., Jennings, P., Bitter, W. \& Vos, J. C. (2019) Function, essentiality, and expression of cytochrome P450 enzymes and their cognate redox partners in Mycobacterium tuberculosis: are they drug targets?, Applied microbiology and biotechnology. 103, 3597-3614.

41. Lee, W. Y., Brune, D. C., LoBrutto, R. \& Blankenship, R. E. (1995) Isolation, characterization, and primary structure of rubredoxin from the photosynthetic bacterium, Heliobacillus mobilis, Archives of biochemistry and biophysics. 318, 80-8.

42. Shumyantseva, V. V., Sigolaeva, L. V., Agafonova, L. E., Bulko, T. V., Pergushov, D. V., Schacher, F. H. \& Archakov, A. I. (2015) Facilitated biosensing via direct electron transfer of myoglobin integrated into diblock copolymer/multi-walled carbon nanotube nanocomposites, Journal of Materials Chemistry B. 3, 5467-5477. 
43. Vasilevskaya, A. V., Yantsevich, A. V., Sergeev, G. V., Lemish, A. P., Usanov, S. A. \& Gilep, A. A. (2017) Identification of Mycobacterium tuberculosis enzyme involved in vitamin D and 7dehydrocholesterol metabolism, The Journal of steroid biochemistry and molecular biology. 169, 202-209.

44. Fischer, F., Raimondi, D., Aliverti, A. \& Zanetti, G. (2002) Mycobacterium tuberculosis FprA, a novel bacterial NADPH-ferredoxin reductase, European journal of biochemistry. 269, 3005-13. 45. Moser, A. \& Korstjens, I. (2018) Series: Practical guidance to qualitative research. Part 3: Sampling, data collection and analysis, The European journal of general practice. 24, 9-18.

46. Kabsch, W. (2010) Xds, Acta Crystallogr D Biol Crystallogr. 66, 125-32.

47. Vagin, A. \& Lebedev, A. (2015) MoRDa, an automatic molecular replacement pipeline, Acta Crystallographica Section A Foundations and Advances. 71, s19-s19.

48. Terwilliger, T. C., Grosse-Kunstleve, R. W., Afonine, P. V., Moriarty, N. W., Zwart, P. H., Hung, L. W., Read, R. J. \& Adams, P. D. (2008) Iterative model building, structure refinement and density modification with the PHENIX AutoBuild wizard, Acta Crystallogr D Biol Crystallogr. 64, 61-9.

49. Murshudov, G. N., Skubak, P., Lebedev, A. A., Pannu, N. S., Steiner, R. A., Nicholls, R. A., Winn, M. D., Long, F. \& Vagin, A. A. (2011) REFMAC5 for the refinement of macromolecular crystal structures, Acta Crystallogr D Biol Crystallogr. 67, 355-67.

50. Afonine, P. V., Grosse-Kunstleve, R. W., Echols, N., Headd, J. J., Moriarty, N. W., Mustyakimov, M., Terwilliger, T. C., Urzhumtsev, A., Zwart, P. H. \& Adams, P. D. (2012) Towards automated crystallographic structure refinement with phenix.refine, Acta Crystallogr D Biol Crystallogr. 68, 352-67.

51. Emsley, P., Lohkamp, B., Scott, W. G. \& Cowtan, K. (2010) Features and development of Coot, Acta Crystallogr D Biol Crystallogr. 66, 486-501.

52. Williams, C. J., Headd, J. J., Moriarty, N. W., Prisant, M. G., Videau, L. L., Deis, L. N., Verma, V., Keedy, D. A., Hintze, B. J., Chen, V. B., Jain, S., Lewis, S. M., Arendall, W. B., 3rd, Snoeyink, J., Adams, P. D., Lovell, S. C., Richardson, J. S. \& Richardson, D. C. (2018) MolProbity: More and better reference data for improved all-atom structure validation, Protein Sci. 27, 293-315.

53. Touw, W. G., Baakman, C., Black, J., te Beek, T. A., Krieger, E., Joosten, R. P. \& Vriend, G. (2015) A series of PDB-related databanks for everyday needs, Nucleic Acids Res. 43, D364-8.

54.

55. Zheng, H., Cooper, D. R., Porebski, P. J., Shabalin, I. G., Handing, K. B. \& Minor, W. (2017) CheckMyMetal: a macromolecular metal-binding validation tool, Acta Crystallogr D Struct Biol. 73, 223-233.

56. Foley, G., Sutzl, L., D'Cunha, S. A., Gillam, E. M. \& Boden, M. (2019) SeqScrub: a web tool for automatic cleaning and annotation of FASTA file headers for bioinformatic applications, Biotechniques. 67, 50-54.

57. Larkin, M. A., Blackshields, G., Brown, N. P., Chenna, R., McGettigan, P. A., McWilliam, H., Valentin, F., Wallace, I. M., Wilm, A., Lopez, R., Thompson, J. D., Gibson, T. J. \& Higgins, D. G. (2007) Clustal W and Clustal X version 2.0, Bioinformatics. 23, 2947-8. 
Table 1. Influence of $\mathrm{pH}$ on thermal stability of RubB estimated using DSC

\begin{tabular}{|c|cccccc|}
\hline $\mathrm{pH}$ & 2.0 & 3.0 & 4.0 & 5.0 & 6.0 & 7.4 \\
\hline $\mathrm{T}_{\mathrm{m}},{ }^{\circ} \mathrm{C}$ & $57.4 \pm 0.6$ & $60.4 \pm 0.8$ & $70.1 \pm 0.9$ & $87.2 \pm 0.7$ & $\mathrm{nd}$ & $\mathrm{nd}$ \\
\hline
\end{tabular}

nd $-\mathrm{T}_{\mathrm{m}}$ could not be determined

Table 2. Electrochemical parameters of RubB, FprA, and FdR.

Analyses were performed on an SPE modified with MWCNT in 0.1 M potassium phosphate buffer ( $\mathrm{pH}$ 7.4), containing $0.05 \mathrm{M} \mathrm{NaCl}$. $\mathrm{E}_{\mathrm{pc}}$ is the signal at the cathodic peak, $\mathrm{E}_{\mathrm{pa}}$ is the signal at the anodic peak, and $\mathrm{E}^{0}$ is the midpoint potential.

\begin{tabular}{|c|c|c|c|}
\hline & $\begin{array}{l}\mathrm{E}_{\mathrm{pc}} \\
\mathrm{mV}\end{array}$ & $\begin{array}{l}\mathrm{E}_{\mathrm{pa}}, \\
\mathrm{mV}\end{array}$ & $\begin{array}{c}\text { The midpoint } \\
\text { potential } \mathrm{E}^{0^{1}} \text {, } \\
\mathrm{mV}\end{array}$ \\
\hline RubB & $-356 \pm 6$ & $-173 \pm 11$ & $-264 \pm 17$ \\
\hline FprA & $-688 \pm 20$ & $-581 \pm 30$ & $-625 \pm 50$ \\
\hline FdR & $-645 \pm 31$ & $-594 \pm 31$ & $-619 \pm 62$ \\
\hline
\end{tabular}


Table 3. Enzymatic activity of CYPs in the reconstituted system containing RubB and reductases.

If RubB alone or reductase alone were added to CYP and $\mathrm{NAD}(\mathrm{P}) \mathrm{H}$, catalytic activity was not detected. CYP124 catalytic activity was measured by oxidation of 7-ketocholesterol. CYP125 and CYP142 activities were measured by oxidation of cholestenone.

\begin{tabular}{|l|lll|}
\hline \multicolumn{1}{|c|}{ Reconstituted system } & \multicolumn{3}{|c|}{ Enzymatic activity, min $^{-\mathbf{1}}$} \\
& CYP124 & CYP125 & CYP142 \\
\hline Arh1 (from Saccharomyces cerevisiae), RubB & $4.23 \pm 0.06$ & $0.36 \pm 0.04$ & $1.08 \pm 0.01$ \\
FprA (from Mtb), RubB & & & \\
FdR (from Mtb), RubB & $2.35 \pm 0.16$ & $0.52 \pm 0.02$ & $0.98 \pm 0.20$ \\
PETH (from Spinacia oleracea), RubB & & & \\
& $2.49 \pm 0.03$ & $0.09 \pm 0.03$ & $0.48 \pm 0.11$ \\
& & & \\
& $5.25 \pm 0.42$ & $0.56 \pm 0.30$ & $1.01 \pm 0.09$ \\
\hline
\end{tabular}

Table 4. Effect of $\mathrm{Zn}^{2+}$ on catalytic activity of CYP124.

\begin{tabular}{|l|cccccc|c|}
\hline & \multicolumn{5}{|c|}{$\mathrm{Zn}^{2+}, \mathrm{mM}$} & & $\mathrm{Ni}^{2+}$, \\
& 0 & 0.1 & 0.5 & 1 & 10 & 10 \\
\cline { 2 - 7 } & 0 & & & & & \\
\hline $\begin{array}{l}\text { Enzymatic activity, } \\
\text { \% of substrate } \\
\text { conversion }\end{array}$ & 50.1 & 0.3 & 0.3 & 0.4 & 0 & 25.4 \\
\hline
\end{tabular}


Table 5. Influence of zinc on thermal stability of RubB.

\begin{tabular}{|ccc|}
\hline $\mathrm{Zn}^{2+}$ concentration, & $\mathrm{T}_{\mathrm{m} 1,}$ & $\mathrm{~T}_{\mathrm{m} 2,}$ \\
$\mathrm{mM}$ & ${ }^{\circ} \mathrm{C}$ & ${ }^{\circ} \mathrm{C}$ \\
\hline 1 & 54.7 & 87.4 \\
5 & 62.4 & 93.4 \\
10 & 65.7 & 98.0 \\
\hline
\end{tabular}

Table 6. Thermodynamic parameters for RubB binding to CYP124.

\begin{tabular}{|l|cccc|}
\hline & & & & \\
& $\mathrm{K}_{\mathrm{d}}$, & $\Delta \mathrm{H}$, & $-\mathrm{T} \Delta \mathrm{S}$, & $\Delta \mathrm{G}$, \\
& $\mu \mathrm{M}$ & $\mathrm{kcal} / \mathrm{mol}$ & $\mathrm{kcal} / \mathrm{mol}$ & $\mathrm{kcal} / \mathrm{mol}$ \\
\hline CYP124 & $12.8 \pm 0.7$ & $-1.8 \pm 0.2$ & $-4.5 \pm 0.1$ & $-6.3 \pm 0.1$ \\
CYP124 plus cholestenone & $20.3 \pm 1.2$ & $-1.7 \pm 0.1$ & $-4.5 \pm 0.1$ & $-6.2 \pm 0.1$ \\
CYP124 plus 0.15 M NaCl & $61.8 \pm 3.5$ & $-29 \pm 3.0$ & $23.5 \pm 1.9$ & $-5.5 \pm 0.2$ \\
\hline
\end{tabular}


Table 7. Thermodynamic parameters for wild type and mutant RubB. Thermal stability was determined by DSC in $20 \mathrm{mM}$ citrate buffer, $\mathrm{pH} 5.0$.

\begin{tabular}{|ccc|}
\hline RubB & Tm, & $\Delta \mathrm{H}$, \\
& ${ }^{\circ} \mathrm{C}$ & $\mathrm{kcal} / \mathrm{mol}$ \\
\hline Wild type & 87.2 & 119.8 \\
E24A & 87.9 & 117.8 \\
D44A & 92.7 & 120.1 \\
D25A & 86.9 & 118.2 \\
& & 117.5 \\
S41V & 88.9 & 122.6 \\
\hline Q11V & 89.6 & \\
\hline
\end{tabular}


Fig. 1. UV-Vis absorbance spectrum of RubB.

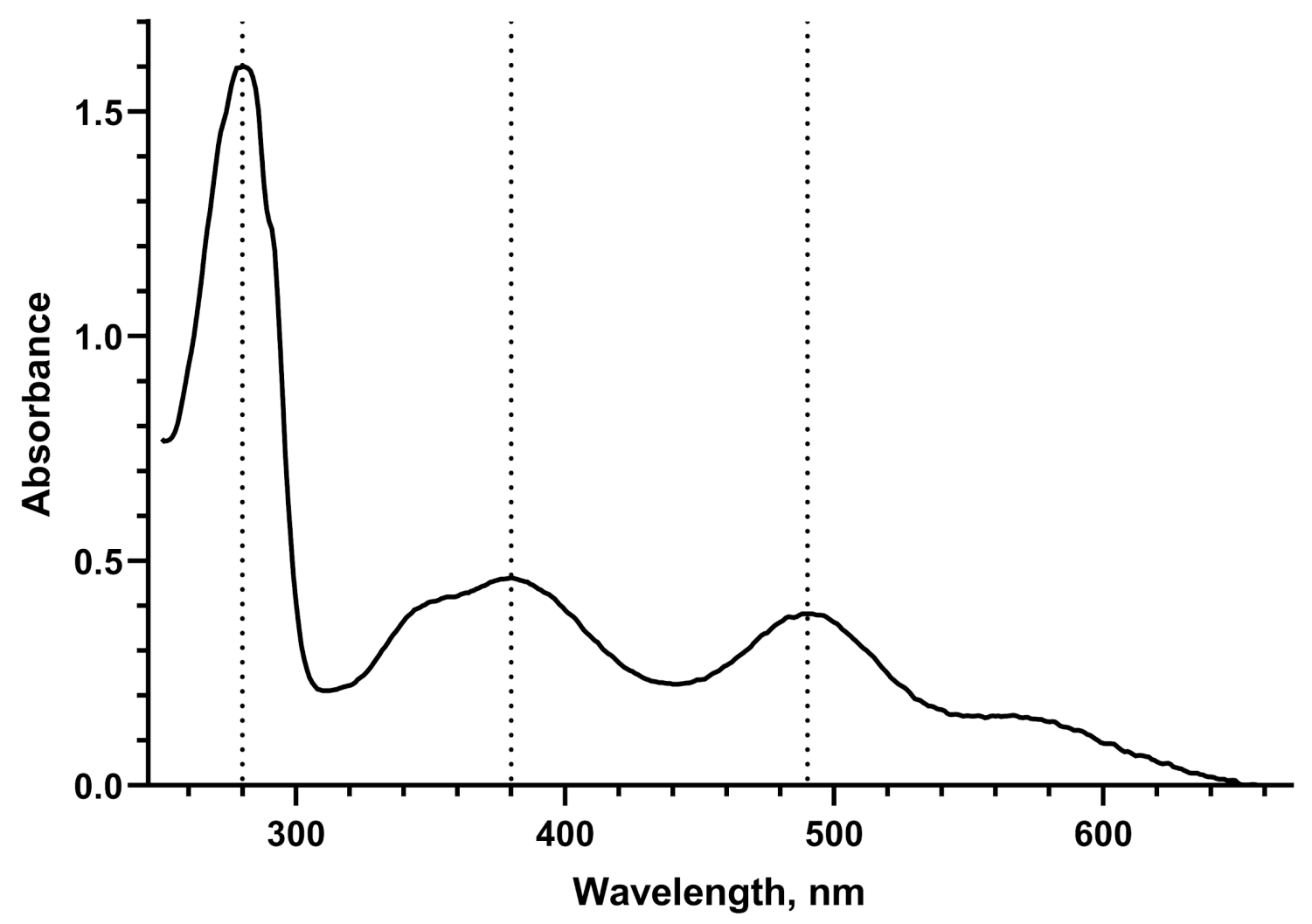


Fig 2. (A) Direct non-catalytic cyclic voltammograms of MWCNT-modified SPE without (solid lines) and with RubB (dashed lines) in $0.1 \mathrm{M}$ potassium phosphate buffer ( $\mathrm{pH} 7.4$ ) containing 0.05 $\mathrm{M} \mathrm{NaCl}$ from 50 to $100 \mathrm{mV} / \mathrm{s}$. (B) Dependence of cathodic and anodic current on scan rate.

A

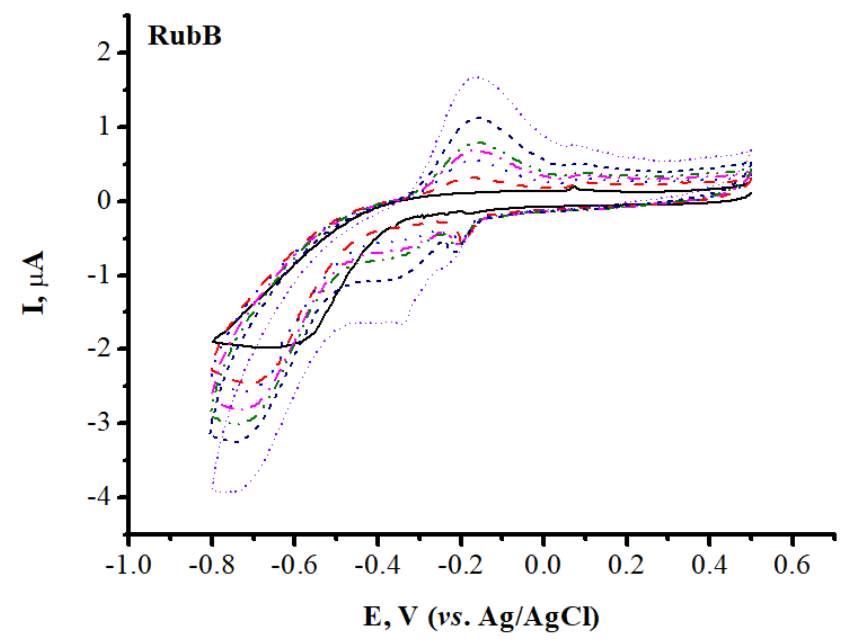

B

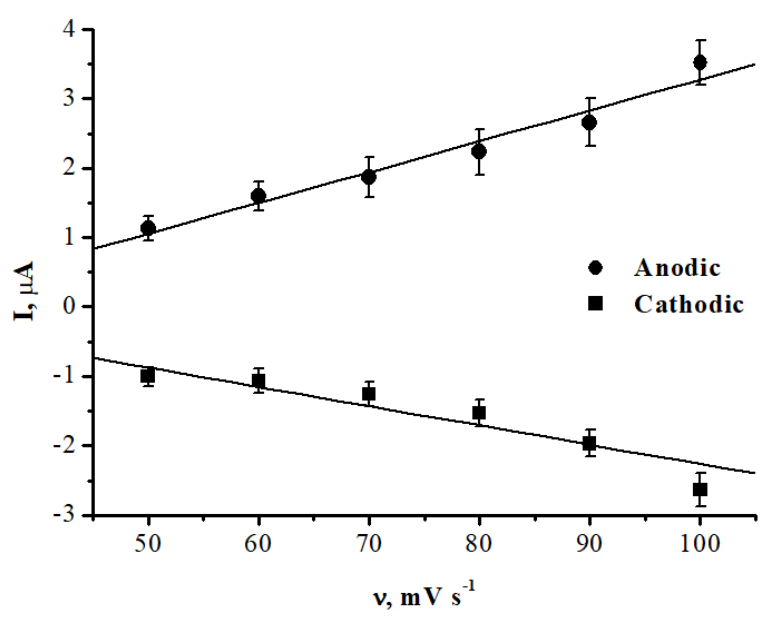


Fig 3. Structures of the eight monomers of RubB in the asymmetric unit of the crystal structure. Aligned structures of $\mathrm{C} \alpha$ bonds are shown as sticks. The central $\beta$-sheet is colored blue, the $31^{-}$ helices (denoted A, B and C) are colored red. In the $\mathrm{FeS}_{4}$ cluster, the iron ion is shown as an orange sphere, and sulfurs of the coordinating cysteines are depicted in yellow. The differences between monomers in the asymmetric unit are determined to a great extent by M1 at the N-terminus and E56-S60 at the C-terminus, for which average pairwise RMSD is $4.95 \AA$; for other residues pairwise RMSDs do not exceed $0.44 \AA$. This result is consistent with previously solved NMR structure, where residues D3-E56 had $0.39 \AA$ RMSD for backbone atoms.

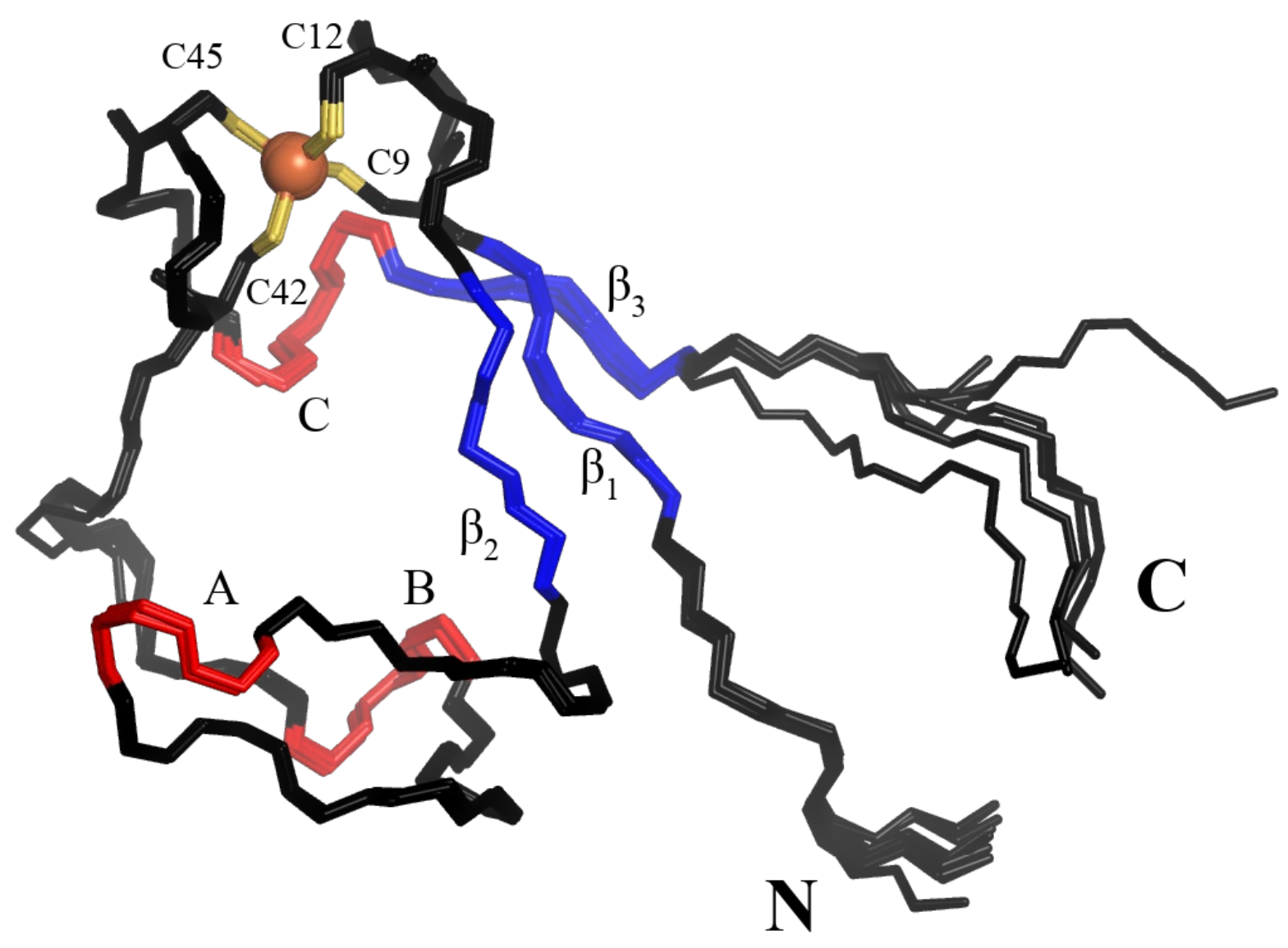


Fig 4. Catalytic activity of CYP124 reconstituted with RubB mutants in the system with Arh1 (blue bars), FprA (red bars), or FdR (grey bars).

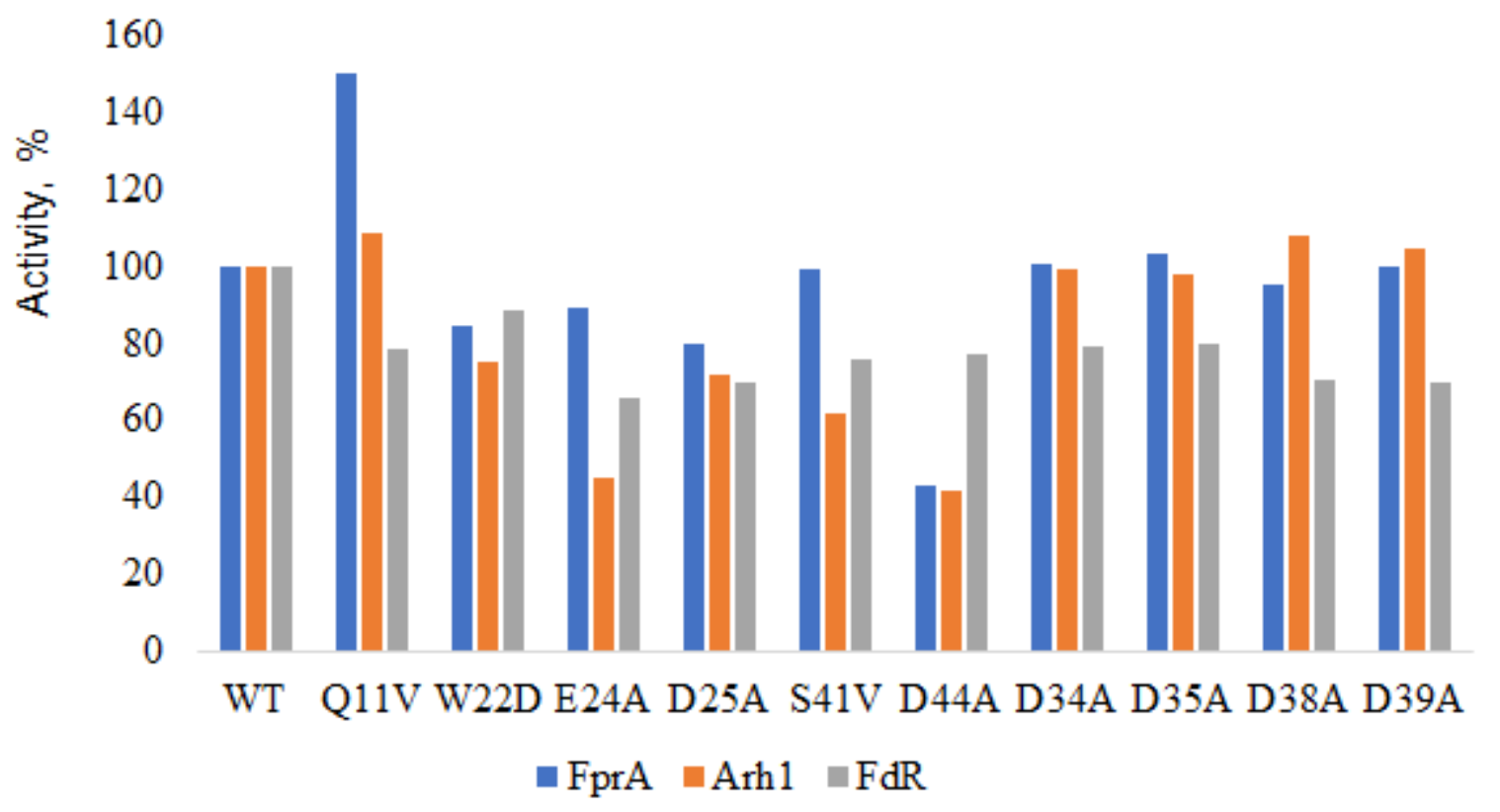


Fig 5. Catalytic activity of CYP125 (blue bars) and CYP142 (orange bars) reconstituted with FprA and indicated RubB mutants.

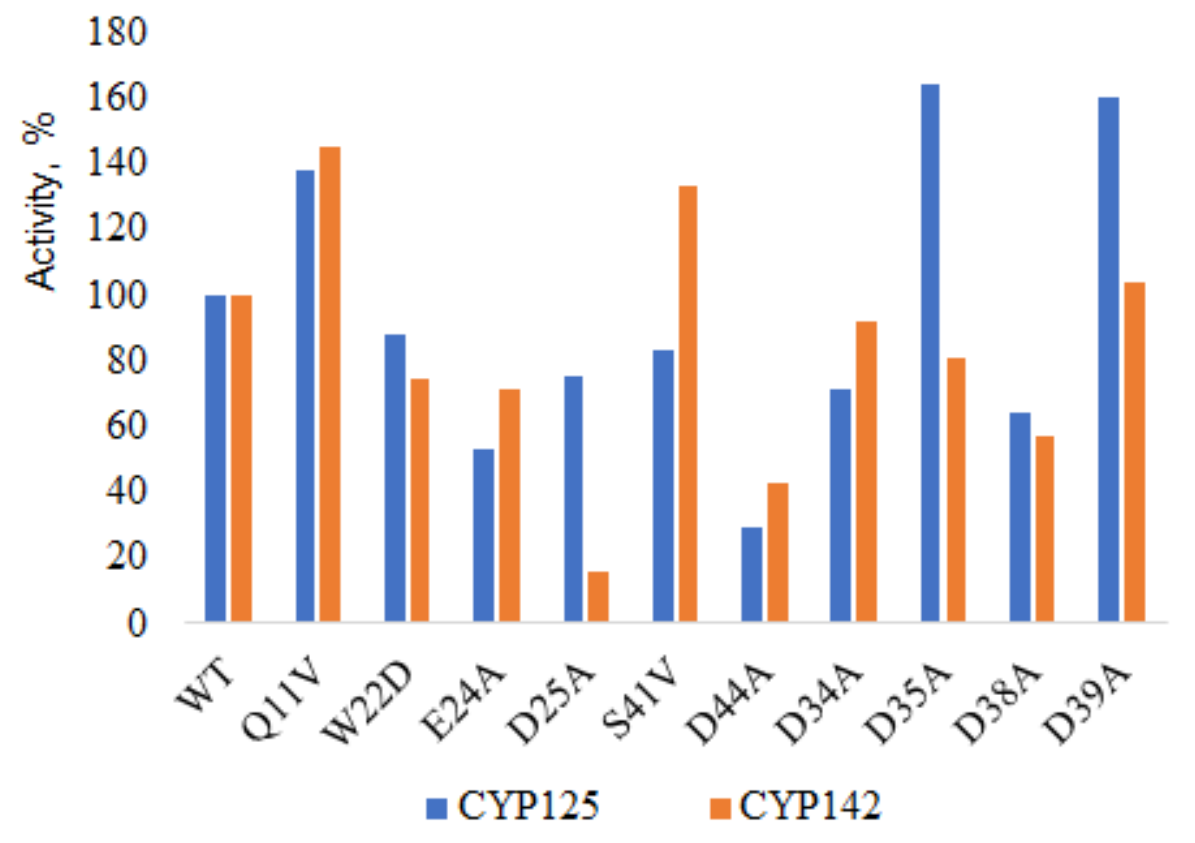


Fig 6. Electrostatic potential on the proximal surface of CYP124 (a), CYP125 (b), CYP142 (c) and RubB (d).
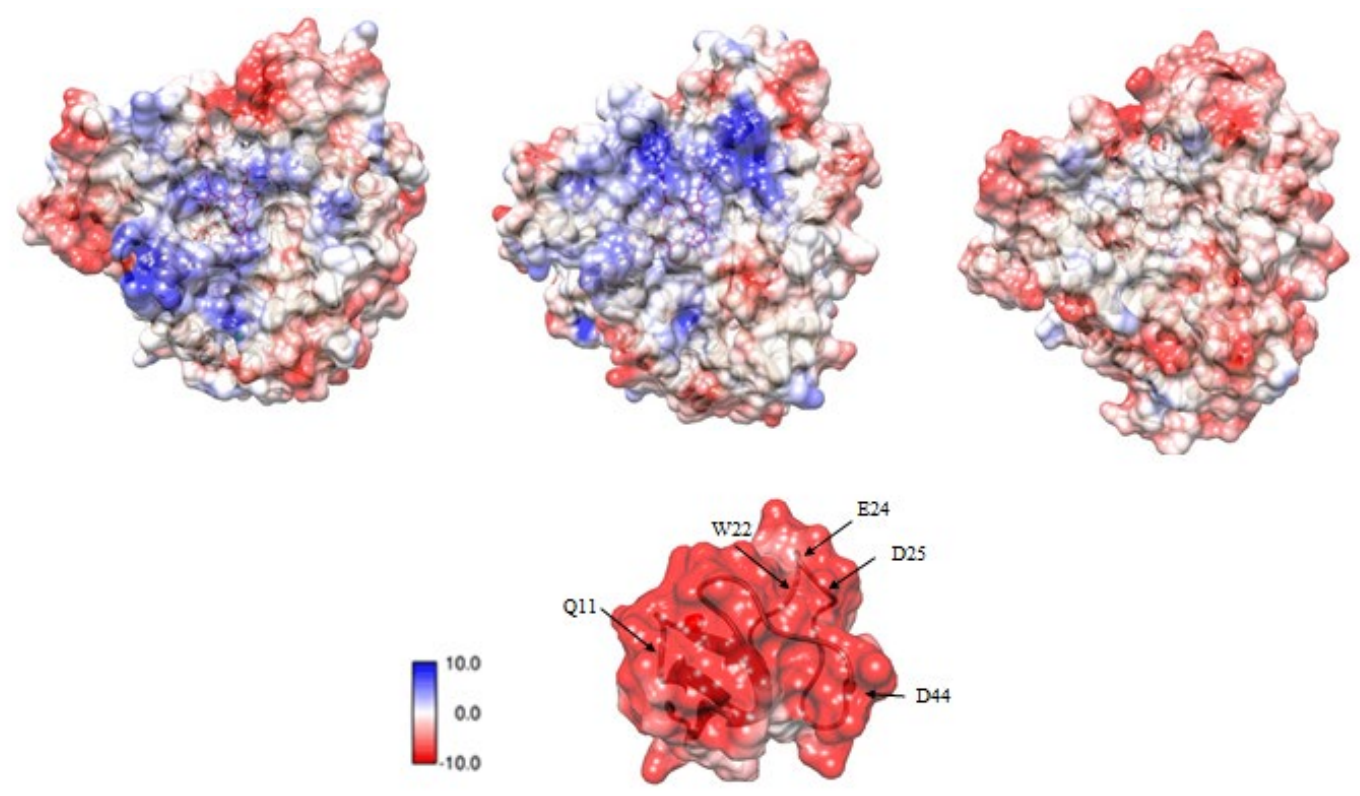